\title{
Pathophysiology of Potassium-competitive Acid Blocker-refractory Gastroesophageal Reflux and the Potential of Potassium-competitive Acid Blocker Test
}

\author{
Tatsuhiro Masaoka, ${ }^{\text {* }}$ Hisako Kameyama, ${ }^{1}$ Tsuyoshi Yamane, ${ }^{1}$ Yuta Yamamoto,, ${ }^{1}$ Hiroya Takeuchi, ${ }^{2,3}$ Hidekazu Suzuki, \\ Yuko Kitagawa, ${ }^{2}$ and Takanori Kanai ${ }^{1}$ \\ ${ }^{I}$ Division of Gastroenterology and Hepatology, Department of Internal Medicine, Keio University School of Medicine, Tokyo, Japan; ${ }^{2}$ Department \\ of Surgery, Keio University School of Medicine, Tokyo, Japan; ${ }^{3}$ Department of Surgery, Hamamatsu University School of Medicine, Shizuoka, \\ Japan; and ${ }^{4}$ Medical Education Center, Keio University School of Medicine, Tokyo, Japan
}

\section{Background/Aims}

Potassium-competitive acid blockers are expected to be the next generation of drugs for the treatment of diseases caused by gastric acid. In 2015, vonoprazan fumarate, a novel potassium-competitive acid blocker, was approved by the Japanese health insurance system. Since its approval, patients refractory to vonoprazan can be encountered in clinical settings. We designed this study to clarify the pathophysiology of gastroesophageal reflux disease refractory to vonoprazan.

\section{Methods}

In this retrospective study, we involved patients who had refractory symptoms after administration of standard-dose proton pump inhibitors or vonoprazan and underwent diagnostic testing with esophageal high-resolution manometry and 24-hour multichannel intraluminal impedance and pH monitoring while using proton pump inhibitors or vonoprazan. Patients were diagnosed based on the Rome IV criteria for functional gastrointestinal disorders and diagnostic test results.

\section{Results}

Twenty-seven patients were analyzed during this study. Gastric $\mathrm{pH} \geq 4$ was sustained for a longer period of time, and the esophageal acid exposure time and number of acid reflux events were shorter in the vonoprazan group than in the proton pump inhibitor group. The percentage of patients diagnosed with acidic gastroesophageal reflux disease in the vonoprazan group was lower than that in the proton pump inhibitor group.

\section{Conclusions}

Intra-gastric $\mathrm{pH}$ and acid reflux were strongly suppressed by $20-\mathrm{mg}$ vonoprazan. When patients with gastroesophageal reflux disease present symptoms after administration of 20-mg vonoprazan, the possibility of pathophysiologies other than acid reflux should be considered.

(J Neurogastroenterol Motil 2018;24:577-583)

Key Words

Esophageal motility disorders; Esophageal pH monitoring; Gastroesophageal reflux; Proton pump inhibitors

Received: March 3, 2018 Revised: June 8, 2018 Accepted: June 17, 2018

(a) This is an Open Access article distributed under the terms of the Creative Commons Attribution Non-Commercial License (http://creativecommons. org/licenses/by-nc/4.0) which permits unrestricted non-commercial use, distribution, and reproduction in any medium, provided the original work is properly cited.

*Correspondence: Tatsuhiro Masaoka, MD, PhD

Division of Gastroenterology and Hepatology, Department of Internal Medicine, Keio University School of Medicine, 35 Shinanomachi, Shinjuku-ku, Tokyo 160-8582, Japan

Tel: +81-3-5363-3790, Fax: +81-3-3353-6247, E-mail: masaoka@keio.jp 


\section{Introduction}

Gastroesophageal reflux disease (GERD) is a condition in which the reflux of gastric contents causes esophageal mucosal damage, uncomfortable symptoms, or both. ${ }^{1}$ A recent systematic review of the epidemiology of GERD reported a prevalence of 20$30 \%$ in Western countries, and this prevalence is increasing, particularly in North America and East Asia. ${ }^{2}$ Physiologically, the main component of gastric juice is hydrochloric acid. Therefore, acidsuppressive therapy is the standard medical treatment for GERD. Historically, acid-suppressive therapy has started with the administration of antacids and anticholinergics. In the 1970s, histamine H2 receptor antagonists were mainly used. Since the 1980s, proton pump inhibitors (PPIs) have been the major acid-suppressive therapy used in clinical practice.

Although PPI therapy is highly effective, a subset of patients has GERD that is refractory to PPI therapy and is referred to as refractory GERD. The pathophysiology of refractory GERD is considered heterogeneous. This condition could be the result of an incorrect diagnosis of GERD, inadequate PPI intake, persistent acid reflux, or non-acidic reflux. ${ }^{3}$ Moreover, approximately onethird of patients with refractory reflux symptoms were reported to have esophageal disorders other than GERD. ${ }^{4}$

The development of new drugs such as extended-release PPIs, combination PPIs, transient lower esophageal sphincter relaxation (LES) reducers, and esophageal pain modulators has aimed to improve the outcomes of GERD therapy. However, to date, no drug has been more effective than the currently available treatments. ${ }^{5}$ Potassium $\left(\mathrm{K}^{+}\right)$-competitive acid blockers (P-CABs) reversibly inhibit gastric $\mathrm{H}^{+} / \mathrm{K}^{+}$-ATPase by competing with $\mathrm{K}^{+}$. The pharmacological effects of $\mathrm{P}-\mathrm{CABs}$ include fast onset, high magnitudes, and long durations. Therefore, P-CABs were thought to represent the next generation of drugs for gastric acid-related diseases. However, attempts to market P-CABs have failed, presumably for reasons such as study design or adverse effects such as liver toxicity. The only successfully marketed P-CAB in Korea was revaprazan. ${ }^{5}$ In 2015, vonoprazan fumarate (TAK-438), a novel P-CAB, was approved for the treatment of gastric acid-related diseases such as peptic ulcers, GERD, and Helicobacter pylori infection by the Japanese health insurance system. Compared with PPI-based (lansoprazole or rabeprazole) triple therapy, the efficacy of vonoprazan-based triple therapy was reported to be superior. ${ }^{6,7}$ The efficacy after switching to vonoprazan for patients with PPI-resistant reflux esophagitis was also reported.
Two years since its approval, patients with GERD refractory to vonoprazan have been encountered in clinical settings. We designed this study to clarify the pathophysiology of GERD refractory to PCABs.

\section{Materials and Methods}

\section{Patients}

Patients who presented refractory symptoms that were presumed to be reflux-related (heartburn, regurgitation, retrosternal pain, and laryngeal discomfort) after using a standard-dose PPI (30-mg lansoprazole, 20-mg rabeprazole, or 20-mg esomeprazole) or a standard-dose P-CAB (20-mg vonoprazan) for more than 8 weeks, and who underwent esophageal pathophysiological evaluation by both 24-hour multichannel intraluminal impedance and $\mathrm{pH}$ (24-hour MII-pH) monitoring and esophageal high-resolution manometry (HRM) from September 2014 to August 2017 were involved in the study. When patients were switched from a PPI to $\mathrm{P}-\mathrm{CAB}$, the P-CAB treatment was longer than 4 weeks. Before or after referral to our hospital, all included patients underwent esophagogastroduodenoscopy (EGD) to check for the presence of mucosal damage and the development of a hiatal hernia during the treatment. When EGD revealed reflux esophagitis, the findings were classified according to the Los Angeles classification. ${ }^{9} \mathrm{H}$. $\mathrm{py}$ lori infection was evaluated using one or more methods which were H. pylori cultures obtained from gastric biopsy specimens, a stool antigen test, and a urea breath test. Concomitance of functional dyspepsia was diagnosed using the Rome IV criteria. ${ }^{10}$ The frequency and severity of GERD-related symptoms were evaluated using a validated questionnaire called the GerdQ. ${ }^{11}$ Patients diagnosed with achalasia were excluded from the analysis. Patients who had a complicated peptic ulcer or malignant tumor were also excluded. Informed consent was obtained by the opt-out method. This retrospective study was approved by the Ethics Committee of the Keio University School of Medicine (No. 20150081).

\section{Esophageal High-resolution Manometry}

The Starlet HRM system (Star Medical, Inc, Tokyo, Japan) with a 36-channel solid-state catheter (Unisensor AG, Attikon, Switzerland) was used for HRM. ${ }^{12}$ Before insertion of the catheter, patients were fasted for at least 12 hours. After local anesthesia with $2 \%$ viscous xylocaine (AstraZeneca K.K., Osaka, Japan) was given, physicians transnasally inserted the catheter. HRM was performed according to the protocol recommended by the Chicago classifica- 
tion (version 3.0). ${ }^{13}$ Physicians analyzed the obtained data with specialized software for the system (Star Medical, Inc). Esophageal motility disorders were classified based on the Chicago classification (version 3.0). Based on a previous report, the cut-off value of impaired LES relaxation was defined as integrated relaxation pressure $\geq 15 \mathrm{mmHg}$.

\section{Monitoring of 24-hour Multichannel Intraluminal Impedance and $\mathrm{pH}$}

Patients underwent 24-hour MII-pH monitoring while using a standard-dose PPI (30-mg lansoprazole, 20-mg rabeprazole, or 20mg esomeprazole) or standard-dose P-CAB (20-mg vonoprazan). Before insertion of the catheter, patients took the prescribed PPI or $\mathrm{P}-\mathrm{CAB}$. Before insertion of a combined $\mathrm{pH}$-impedance catheter (model ZAN-BG-44; Sandhill Scientific Inc, Highlands Ranch, CO, USA), patients were fasted for at least 12 hours. Calibration of the electrodes for $\mathrm{pH}$ measurement positioned on the catheter was done with 2 buffer solutions ( $\mathrm{pH} 4.0$ and $\mathrm{pH}$ 7.0) before insertion of the catheter. After local anesthesia with $2 \%$ viscous xylocaine, physicians transnasally inserted the catheter. The $\mathrm{pH}$ electrodes for gastric $\mathrm{pH}$ measurements were positioned $10 \mathrm{~cm}$ below the LES. The $\mathrm{pH}$ electrodes for esophageal $\mathrm{pH}$ measurements were positioned $5 \mathrm{~cm}$ above the LES. The position of the probe was confirmed through fluoroscopy because HRM and 24-hour MII-pH were not always performed on the same day. The 24-hour MII-pH was performed at least 8 weeks after drug treatment.

Impedance and $\mathrm{pH}$ were recorded with an ambulatory recorder (Sleuth ZepHr; Sandhill Scientific Inc) which was connected to the catheter. During measurements, patients were hospitalized and encouraged to maintain their normal daily activities. Furthermore, they were instructed to press the event marker on the data acquisition unit when they ate, changed body positions, and experienced symptoms. After completion of the recording, physicians analyzed the recorded data with BioView software version 5.6.3.0 (Sandhill Scientific Inc). For confirmation of detection and classification of reflux episodes, data were also manually reviewed by physicians. A decrease in $\mathrm{pH}$ from $>4$ to $<4$ was defined as acid reflux. No change in $\mathrm{pH}$ or a decrease of $<1 \mathrm{pH}$ unit was defined as nonacid reflux. Recorded data during meals were excluded from the analysis.

\section{Data Analysis}

The esophageal total acid exposure time (AET) was defined as the total percentage of time during which the $\mathrm{pH}$ of the distal esophagus was $<4$. When the AET was greater than $6 \%$, patients were considered to have acidic GERD. ${ }^{14}$ The percentage of time during which the gastric $\mathrm{pH}$ was $>4$, reflux-associated symptoms, number of reflux episodes, symptom index (SI), and symptom association probability (SAP) were also recorded. When symptoms occurred within a 2-minute time window for SAP and a 5-minute time window for SI after onset, it was considered as a reflux related episode. SAP $\geq 95 \%$ or $\mathrm{SI} \geq 50 \%$ suggested an association between symptoms and reflux events and was interpreted as positive SI or positive SAP. Patients were diagnosed based on the definition of functional esophageal disorders proposed by Rome ${ }^{15}$ and findings obtained by HRM and 24-hour MII-pH monitoring (Fig. 1). When the symptom frequency during the testing day exceeded 7 events, SI or SAP for the symptom was calculated.

\section{Statistical Methods}

Data are expressed as mean \pm standard deviation. Distributions were tested using the $\mathrm{F}$ test. If distributions were normal, then the Student's $t$ test was used. Otherwise, non-parametric tests (Mann-Whitney $U$ test) were used for analysis. Statistical analysis was performed using BellCurve for Excel (Social Survey Research Information Co, Ltd, Tokyo, Japan). $P<0.05$ was considered significant.

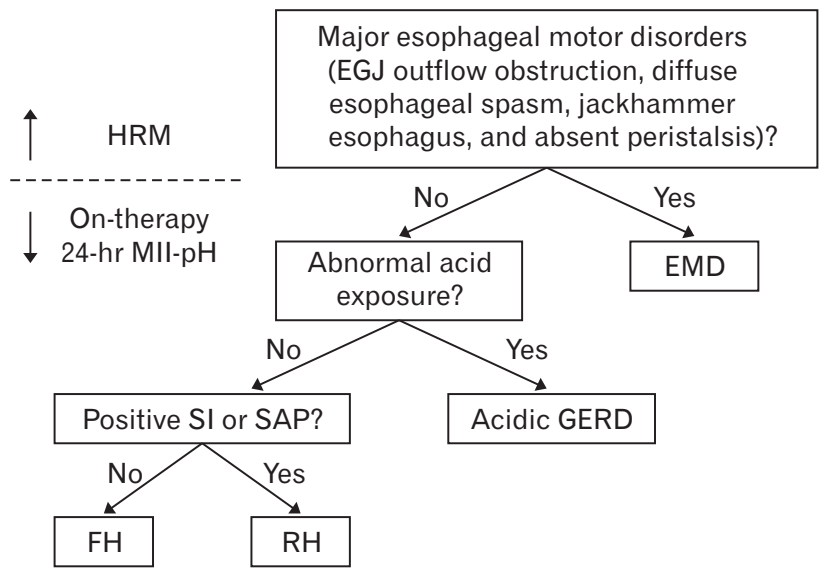

Figure 1. Diagnostic algorithm. HRM, high-resolution manometry; 24-hr MII-pH, 24-hour multichannel intraluminal impedance and pH monitoring; EGJ, esophagogastric junction; EMD, esophageal motility disorder; SI, symptom index; SAP, symptom association probability; GERD, gastroesophageal reflux disease; $\mathrm{FH}$, functional heartburn; RH, reflux hypersensitivity. On-therapy, under potassiumcompetitive acid blocker or proton pump inhibitor treatment. 


\section{Results}

\section{Enrolled Patients}

Thirty patients who presented refractory symptoms that were presumed to be reflux-related after administration of a standarddose PPI or a standard-dose P-CAB underwent both 24-hour MII-pH and esophageal HRM from September 2014 to August 2017 were enrolled. Two patients were excluded from the analysis due to a diagnosis of achalasia. One patient was excluded from the analysis due to a duodenal ulcer diagnosis. Twenty-seven patients (11 in the PPI group and 16 in the P-CAB group) were finally analyzed (Fig. 2). There was no significant difference in clinical background characteristics between the PPI and P-CAB groups. When patients were classified based on findings obtained using EGD, one patient in the PPI group and one patient in the P-CAB group were classified as having grade $\mathrm{B}$ and grade $\mathrm{C}$ reflux esophagitis, respectively. The other patients did not present reflux esophagitis (Table).

\section{Inhibition of Gastric Acid Secretion and Acid Reflux by Potassium-competitive Acid Blockers}

Compared with the PPI group, AET in the P-CAB group was shorter than that in the $\mathrm{P}-\mathrm{CAB}$ group $(P<0.05$; Fig. 3A). Compared with the PPI group, gastric $\mathrm{pH} \geq 4$ was sustained for a longer period of time in the $\mathrm{P}-\mathrm{CAB}$ group $(P<0.05$; Fig. 3B). Acid reflux occurred less frequently in the $\mathrm{P}-\mathrm{CAB}$ group than in the $\mathrm{PPI}$

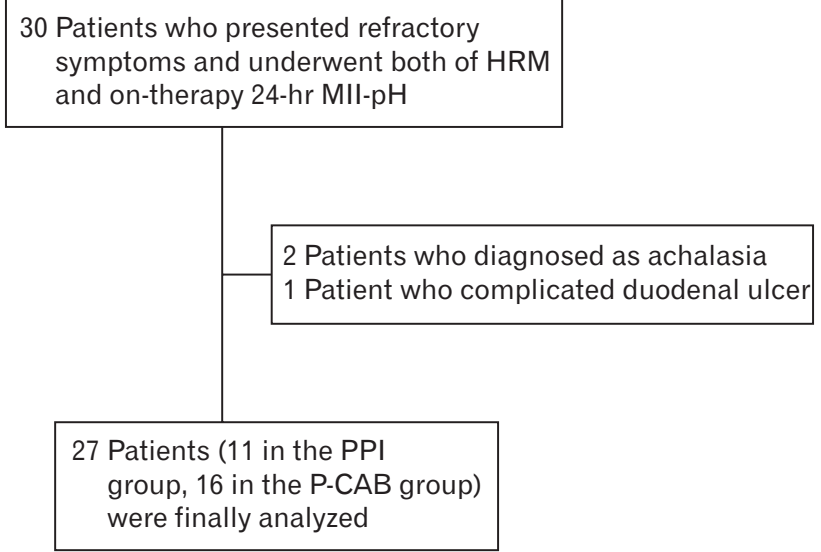

Figure 2. Flow diagram of the study. HRM, high-resolution manometry; 24-hr MII-pH, 24-hour multichannel intraluminal impedance and $\mathrm{pH}$ monitoring; PPI, proton pump inhibitor; $\mathrm{P}-\mathrm{CAB}$, potassiumcompetitive acid blocker. On-therapy, under P-CAB or PPI treatment. group ( $P<0.01$; Fig. 3C). However, non-acid reflux (Fig. 3D) and total reflux (Fig. 3E) occurrences were comparable between groups. P-CAB markedly shortened esophageal acid exposure and inhibited intra-gastric $\mathrm{pH}$ and acid reflux.

\section{Diagnosis Obtained by Esophageal Pathophysiological Evaluation}

Among the enrolled patients, 3 in the PPI group and 1 in the $\mathrm{P}-\mathrm{CAB}$ group had AET $>6 \%$. Although the difference was not statistically significant $(P=0.131)$, the percentage of patients diagnosed with acidic GERD in the P-CAB group was lower than that in the PPI group.

Using HRM, 7 patients were classified as having esophageal motility disorders; 3 (2 with esophagogastric junction outflow obstruction and 1 failed peristalsis) patients were in the PPI group, and 4 patients ( 4 esophagogastric junction outflow obstruction) were in the $\mathrm{P}-\mathrm{CAB}$ group. Based on the definition of functional esophageal disorders proposed by the Rome committee ${ }^{14}$ and findings obtained via on-therapy 24-hour MII-pH monitoring, 5 patients in the $\mathrm{P}-\mathrm{CAB}$ group were classified as having reflux hypersensitivity. The remaining 11 patients ( 5 in the PPI group and 6 in the $\mathrm{P}-\mathrm{CAB}$ group) were classified as having functional heartburn (Fig. 4).

\section{Discussion}

Since the approval of its usage in Japan, the efficacy of vono-

Table. Clinical Characteristics of the Analyzed Patients

\begin{tabular}{|c|c|c|c|}
\hline & $\begin{array}{c}\text { PPI } \\
(n=11)\end{array}$ & $\begin{array}{l}\text { P-CAB } \\
(n=16)\end{array}$ & $P$-value \\
\hline Male/female & $8 / 3$ & $10 / 6$ & 0.580 \\
\hline Age & $57.5 \pm 23.3$ & $58.9 \pm 13.6$ & 0.836 \\
\hline $\mathrm{BMI}$ & $20.3 \pm 3.4$ & $22.6 \pm 4.4$ & 0.163 \\
\hline GerdQ & $8.1 \pm 3.8 \mathrm{a}$ & $9.1 \pm 3.1$ & 0.474 \\
\hline $\begin{array}{l}\text { Presence of } \\
\text { reflux esophagitis }\end{array}$ & $\begin{array}{l}9.1 \%(1 / 11) \\
(\mathrm{LA} \text { grade C) }\end{array}$ & $\begin{array}{l}6.3 \%(1 / 16) \\
\quad(\text { LA grade B) }\end{array}$ & 0.782 \\
\hline $\begin{array}{l}\text { Presence of hiatal } \\
\text { hernia }\end{array}$ & $36.4 \%(4 / 11)$ & $62.5 \%(10 / 16)$ & 0.182 \\
\hline H. pylori infection & $11.1 \%(1 / 9)^{\mathrm{a}}$ & $12.5 \%(2 / 16)$ & 0.918 \\
\hline Concomitant FD & $81.8 \%(9 / 11)$ & $75.0 \%(12 / 16)$ & 0.675 \\
\hline
\end{tabular}

Two patients did not answer the gastroesophageal reflux disease (GerdQ) questionnaire and their Helicobacter pylori (H. pylori) status was not examined because of loss to follow-up.

PPI, proton pump inhibitor; $\mathrm{P}-\mathrm{CAB}$, potassium-competitive acid blocker; BMI, body mass index; LA, Los Angeles classification of reflux esophagitis; FD, functional dyspepsia.

Data are expressed as mean \pm standard deviation. 
A

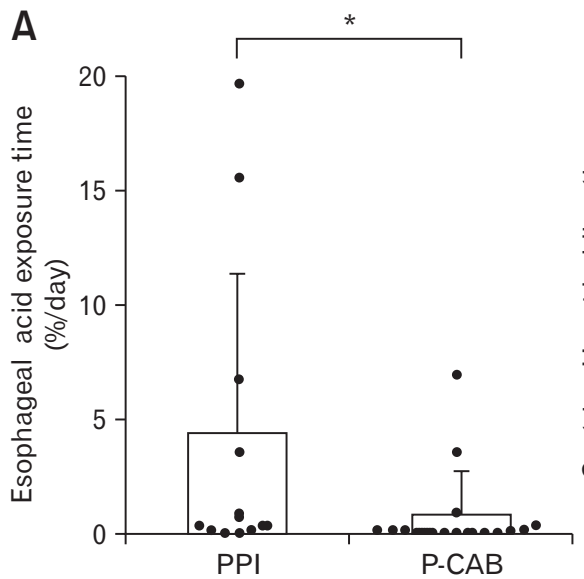

B

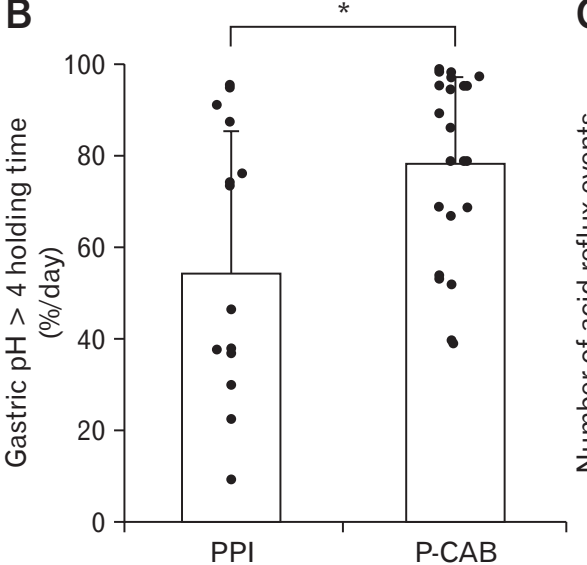

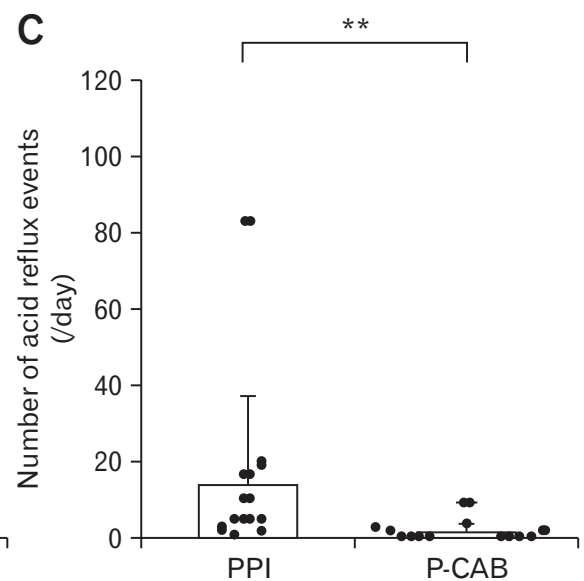

D

$\mathrm{E}$
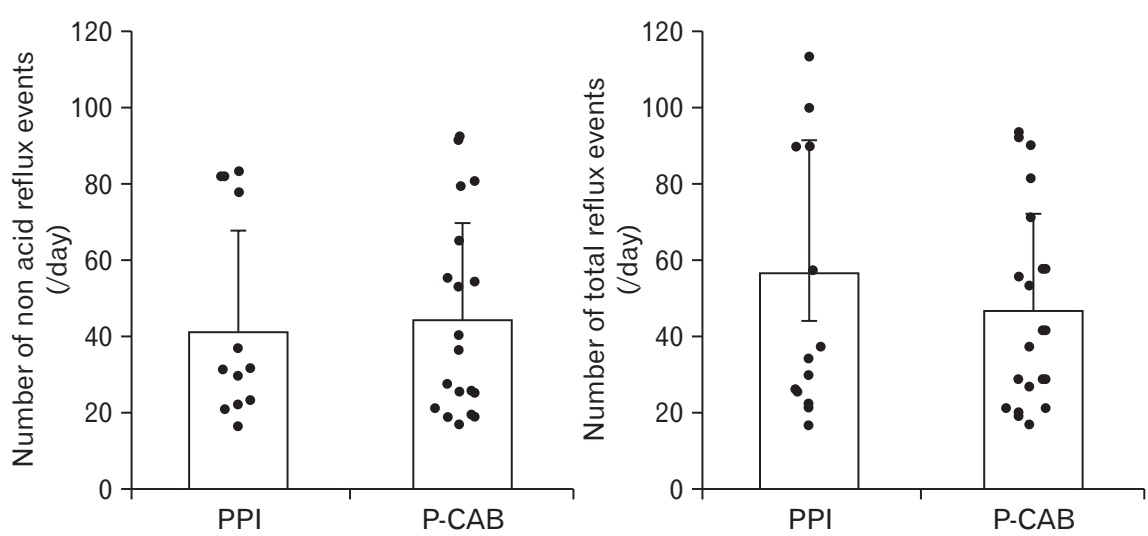

Figure 3. Comparison of parameters obtained by on-therapy 24-hour multichannel intraluminal impedance and pH monitoring. (A) Esophageal acid exposure time. (B) Gastric $\mathrm{pH}>4$ holding time. (C) Number of acid reflux events. (D) Number of non-acid reflux events. (E) Number of total reflux events. ${ }^{*} P<0.05$ and ${ }^{*} P<0.01$. PPI, proton pump inhibitor; P-CAB, potassium-competitive acid blocker.

prazan has been reported for acid-related diseases such as $H$. pylori infection and gastric ulcers ${ }^{7,16}$ and for PPI-resistant reflux esophagitis. ${ }^{17}$ However, to the best of our knowledge, this study is the first to clarify the pathophysiology of P-CAB-refractory GERD assessed by both HRM and MII.

In this study, vonoprazan strongly suppressed gastric $\mathrm{pH}$ and acid reflux. A study involving healthy volunteers demonstrated that gastric $\mathrm{pH} \geq 4$ was maintained for a longer period with vonoprazan. ${ }^{18}$ However, the current study is the first to show shortening of the AET and a reduction in the number of acid reflux incidents experienced by patients with GERD.

In the $\mathrm{P}-\mathrm{CAB}$ group, there was one patient diagnosed with acidic GERD. This patient's gastric $\mathrm{pH}>4$ holding time was $87.5 \%$. On measurement day, there were some periods of time during which the esophageal $\mathrm{pH}$ was $<4$, although the gastric $\mathrm{pH}$ was $>4$. Gastric acid pockets located on the oral side of the gastric
$\mathrm{pH}$ sensor may have contributed to this observation. ${ }^{19}$ Insufficient gastric $\mathrm{pH}$ suppression was not the reason for acidic GERD in this patient.

The prevalence of reflux esophagitis in Japanese GERD patients has been reported to be $41.4 \%{ }^{20}$ Studies have demonstrated that up to $70.0 \%$ of patients with heartburn have normal endoscopy results. ${ }^{21}$ Even for patients with refractory reflux symptoms, the prevalence of acidic GERD confirmed by 24-hour MII-pH was $41.0 \%{ }^{4}$ Compared with previous reports, the rates of reflux esophagitis and acidic GERD in our cohort were low. In the P-CAB group and PPI group, the rate of concomitance with functional dyspepsia was high. The results of this study should be applied only to refractory reflux patients using a PPI or vonoprazan.

Refractory symptoms are generally considered when high-dose PPIs fails. However, the Japanese health insurance system does not support administration of high-dose PPIs. In 2015, the Japanese 


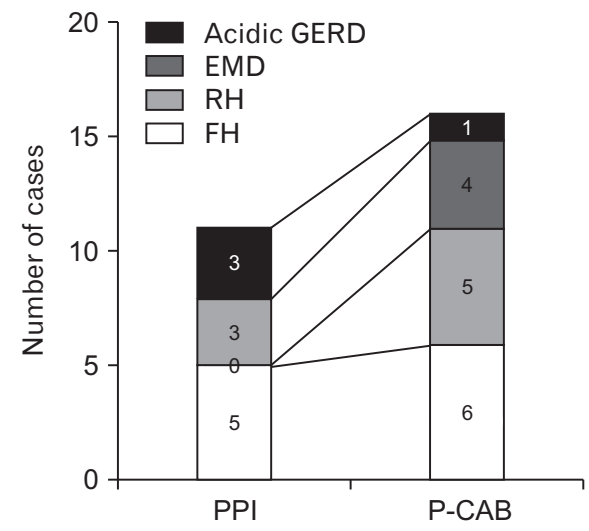

Figure 4. Comparison of diagnoses obtained by esophageal highresolution manometry and on-therapy 24-hour multichannel intraluminal impedance and $\mathrm{pH}$ monitoring in the proton pump inhibitor (PPI) group and potassium-competitive acid blocker (P-CAB) group. Diagnoses of the PPI group and P-CAB group are shown. GERD, gastroesophageal reflux disease; EMD, esophageal motility disorders; $\mathrm{RH}$, reflux hypersensitivity; $\mathrm{FH}$, functional heartburn.

Society of Gastroenterology published evidence-based guidelines for GERD. In the guidelines, the definition of PPI-resistant GERD is as follows: (1) esophageal mucosal break did not heal and/or (2) reflux symptoms considered to be due to GERD were not sufficiently mitigated even after administration of a standard-dose of PPI for 8 weeks. ${ }^{1}$ Therefore, we defined refractory symptoms as failure of a standard-dose PPI.

Esophageal pathophysiological evaluations such as MII and HRM are invasive. However, the PPI test is a well-known noninvasive test for the assessment of GERD. In one meta-analysis using 24-hour $\mathrm{pH}$ monitoring as the gold standard for acid reflux, the sensitivity and specificity of the PPI test were $78.0 \%$ and $54.0 \%$, respectively. ${ }^{22}$ Despite its non-invasiveness, these values are low. Therefore, a new non-invasive test for GERD is necessary. Our results indicated a low prevalence of acidic GERD and strong gastric acid suppression with P-CAB administration. Therefore, the possibility of persistent acid reflux or insufficient gastric acid suppression appears to be low in P-CAB-refractory GERD. The results of this study cannot be generalized to general GERD patients. However, our results suggested that $\mathrm{P}-\mathrm{CAB}$ administration in the form of a P-CAB test could have potential as a new non-invasive test for GERD.

In this study, 20-mg vonoprazan demonstrated a strong acid suppression effect. Many P-CABs before vonoprazan were imidazopyridine-based compounds. However, the chemical structure of vonoprazan differs from that of previous P-CABs. ${ }^{23}$ This different chemical structure can explain the stronger effect of vonoprazan than that of previous P-CABs or PPIs. Therefore, when evaluating the pharmacological effects of vonoprazan, this point should be considered.

This study had several limitations. PPIs are known to be metabolized by cytochrome P450 2C19 (CYP2C19), ${ }^{24}$ and gene polymorphisms of $\mathrm{CYP} 2 \mathrm{C} 19$ were not considered in this study. However, the duration of gastric $\mathrm{pH}>4$ for the PPI group was comparable to that of a previous study. ${ }^{25}$ Moreover, this study was retrospective, conducted at a single-center, and involved a small number of patients. To evaluate the efficacy of the P-CAB test as a new non-invasive test for GERD, prospective multi-center, studies with a larger number of participants should be performed.

In conclusion, this study demonstrated strong inhibition of gastric $\mathrm{pH}$ and acid reflux with administration of 20-mg vonoprazan. Moreover, the prevalence of acidic GERD among cases of PCAB-refractory GERD was low. Although large-scale prospective studies are required, when patients with GERD continue to present symptoms after administration of 20-mg vonoprazan, the possibility of pathophysiologies other than acid reflux should be considered.

Financial support: This study was supported by a grant-in-aid for Scientific Research C (Grant No. 25460301 to Tatsuhiro Masaoka) and Keio Gijuku Academic Development Funds to Tatsuhiro Masaoka.

Conflicts of interest: During the past 2 years, Tatsuhiro Masaoka and Hidekazu Suzuki have received service honoraria from Takeda Pharmaceutical Co Ltd, Otsuka Pharmaceutical Co Ltd, Astra-Zeneca K.K., Daiichi Sankyo Co Ltd, and EA Pharma Co Ltd. Hiroya Takeuchi has received service honoraria from Takeda Pharmaceutical Co Ltd, Daiichi Sankyo Co Ltd, and EA Pharma Co Ltd. Yuko Kitagawa has received service honoraria from AstraZeneca K.K. and Daiichi Sankyo Co Ltd. Takanori Kanai has received service honoraria from Takeda Pharmaceutical Co Ltd, Astra-Zeneca K.K., and EA Pharma Co Ltd.

Author contributions: Tatsuhiro Masaoka designed the study and wrote the initial draft of the manuscript; Hisako Kameyama, Tsuyoshi Yamane, and Yuta Yamamoto contributed to the analysis and interpretation of data; Hiroya Takeuchi, Hidekazu Suzuki, Yuko Kitagawa, and Takanori Kanai contributed to data collection and interpretation and critically reviewed the manuscript; and all authors approved the final version of the manuscript and agreed to be accountable for all aspects of the work by ensuring that questions 
related to the accuracy or integrity of any part of the work are appropriately investigated and resolved.

\section{References}

1. Iwakiri K, Kinoshita Y, Habu Y, et al. Evidence-based clinical practice guidelines for gastroesophageal reflux disease 2015. J Gastroenterol 2016;51:751-767.

2. El-Serag HB, Sweet S, Winchester CC, Dent J. Update on the epidemiology of gastro-oesophageal reflux disease: a systematic review. Gut 2014;63:871-880.

3. Scarpellini E, Ang D, Pauwels A, De Santis A, Vanuytsel T, Tack J. Management of refractory typical GERD symptoms. Nat Rev Gastroenterol Hepatol 2016;13:281-294.

4. Herregods TV, Troelstra M, Weijenborg PW, Bredenoord AJ, Smout AJ. Patients with refractory reflux symptoms often do not have GERD. Neurogastroenterol Motil 2015;27:1267-1273.

5. Maradey-Romero C, Fass R. New and future drug development for gastroesophageal reflux disease. J Neurogastroenterol Motil 2014;20:6-16.

6. Murakami K, Sakurai Y, Shiino M, Funao N, Nishimura A, Asaka M. Vonoprazan, a novel potassium-competitive acid blocker, as a component of first-line and second-line triple therapy for Helicobacter pylori eradication: a phase III, randomised, double-blind study. Gut 2016;65:14391446.

7. Suzuki S, Gotoda T, Kusano C, Iwatsuka K, Moriyama M. The efficacy and tolerability of a triple therapy containing a potassium-competitive acid blocker compared with a 7-day PPI-based low-dose clarithromycin triple therapy. Am J Gastroenterol 2016;111:949-956.

8. Yamashita H, Kanamori A, Kano C, et al. The effects of switching to vonoprazan, a novel potassium-competitive acid blocker, on gastric acidity and reflux patterns in patients with erosive esophagitis refractory to proton pump inhibitors. Digestion 2017;96:52-59.

9. Lundell LR, Dent J, Bennett JR, et al. Endoscopic assessment of oesophagitis: clinical and functional correlates and further validation of the Los Angeles classification. Gut 1999;45:172-180.

10. Stanghellini V, Chan FK, Hasler WL, et al. Gastroduodenal disorders. Gastroenterology 2016;150:1380-1392.

11. Suzuki H, Matsuzaki J, Okada S, Hirata K, Fukuhara S, Hibi T. Validation of the GerdQ questionnaire for the management of gastrooesophageal reflux disease in Japan. United European Gastroenterol J 2013;1:175-183.

12. Bredenoord AJ, Fox M, Kahrilas PJ, et al. Chicago classification criteria of esophageal motility disorders defined in high resolution esophageal pressure topography. Neurogastroenterol Motil 2012;24(suppl 1):57-65.

13. Kahrilas PJ, Bredenoord AJ, Fox M, et al. The Chicago classification of esophageal motility disorders, v3.0. Neurogastroenterol Motil 2015;27:160-174.

14. Roman S, Gyawali CP, Savarino E, et al. Ambulatory reflux monitoring for diagnosis of gastro-esophageal reflux disease: update of the porto consensus and recommendations from an international consensus group. Neurogastroenterol Motil 2017;29:1-15.

15. Aziz Q, Fass R, Gyawali CP, Miwa H, Pandolfino JE, Zerbib F. Functional esophageal disorders. Gastroenterology 2016;150:1368-1379.

16. Tsuchiya I, Kato Y, Tanida E, et al. Effect of vonoprazan on the treatment of artificial gastric ulcers after endoscopic submucosal dissection: prospective randomized controlled trial. Dig Endosc 2017;29:576-583.

17. Hoshino S, Kawami N, Takenouchi N, et al. Efficacy of vonoprazan for proton pump inhibitor-resistant reflux esophagitis. Digestion 2017;95:156-161.

18. Sakurai Y, Mori Y, Okamoto H, et al. Acid-inhibitory effects of vonoprazan $20 \mathrm{mg}$ compared with esomeprazole $20 \mathrm{mg}$ or rabeprazole $10 \mathrm{mg}$ in healthy adult male subjects--a randomised open-label cross-over study. Aliment Pharmacol Ther 2015;42:719-730.

19. Mitchell DR, Derakhshan MH, Robertson EV, McColl KE. The role of the acid pocket in gastroesophageal reflux disease. J Clin Gastroenterol 2016;50:111-119.

20. Fujiwara Y, Arakawa T. Epidemiology and clinical characteristics of GERD in the Japanese population. J Gastroenterol 2009;44:518-534.

21. Yamasaki T, Fass R. Reflux hypersensitivity: a new functional esophageal disorder. J Neurogastroenterol Motil 2017;23:495-503.

22. Numans ME, Lau J, de Wit NJ, Bonis PA. Short-term treatment with proton-pump inhibitors as a test for gastroesophageal reflux disease: a meta-analysis of diagnostic test characteristics. Ann Intern Med 2004;140:518-527.

23. Inatomi N, Matsukawa J, Sakurai Y, Otake K. Potassium-competitive acid blockers: advanced therapeutic option for acid-related diseases. Pharmacol Ther 2016;168:12-22.

24. Ichikawa H, Sugimoto M, Sugimoto K, Andoh A, Furuta T. Rapid metabolizer genotype of CYP2C19 is a risk factor of being refractory to proton pump inhibitor therapy for reflux esophagitis. J Gastroenterol Hepatol 2016;31:716-726.

25. Norris V, Baisley K, Dunn K, Warrington S, Morocutti A. Combined analysis of three crossover clinical pharmacology studies of effects of rabeprazole and esomeprazole on 24-h intragastric $\mathrm{pH}$ in healthy volunteers. Aliment Pharmacol Ther 2007;25:501-510. 